\title{
Evaluation of Spinodal Pressure for Gallium nitride in the Zinc-blend and Wurtzite Structures by Using Different Equations of State $\left(\operatorname{EOS}_{\mathrm{s}}\right)$
}

\author{
*Salar A. Mawlood \\ *Adnan. M. AL-Sheikh \\ ** Mumtaz M. Hussien \\ * Department of Physics / College of Science / University of Mosul \\ **Department of Physics / College of Education for Pure Science / University of Mosul \\ E-mail: salarscience70@hotmail.com E-mail: adnan_alshek E-mail: momtaz_hussien
}

(Received 24/2/2014; Accepted 5/5/2014)

\begin{abstract}
According to the definition of spinodal pressure as the negative pressure at which bulk modulus (of a substance) go to zero. Extrapolation of variation of bulk modulus results with pressure has been used to evaluate spinodal pressure for $\mathrm{GaN}$ in zinc-blende and wurtzite structures using different equations of state (EOS) (Birch-Murnaghan, Bardeen, Libby and Libby, and BornMie). Result obtained in the present work for $\mathrm{GaN}$ in the zinc-blende structure using Born-Mie equation of state shows a good agreement with literature. While results for wurtzite structure, obtained by using Birch-Murnaghan and Bardeen EOSs are in a good agreement with literature. Present results and many literature show that (Jiuxun, 2005) approach for evaluation of spinodal pressure yield results in less agreement with other works.
\end{abstract}

Keywords: EOS, GaN, Spinodal pressure, Negative pressure, bulk modulus.

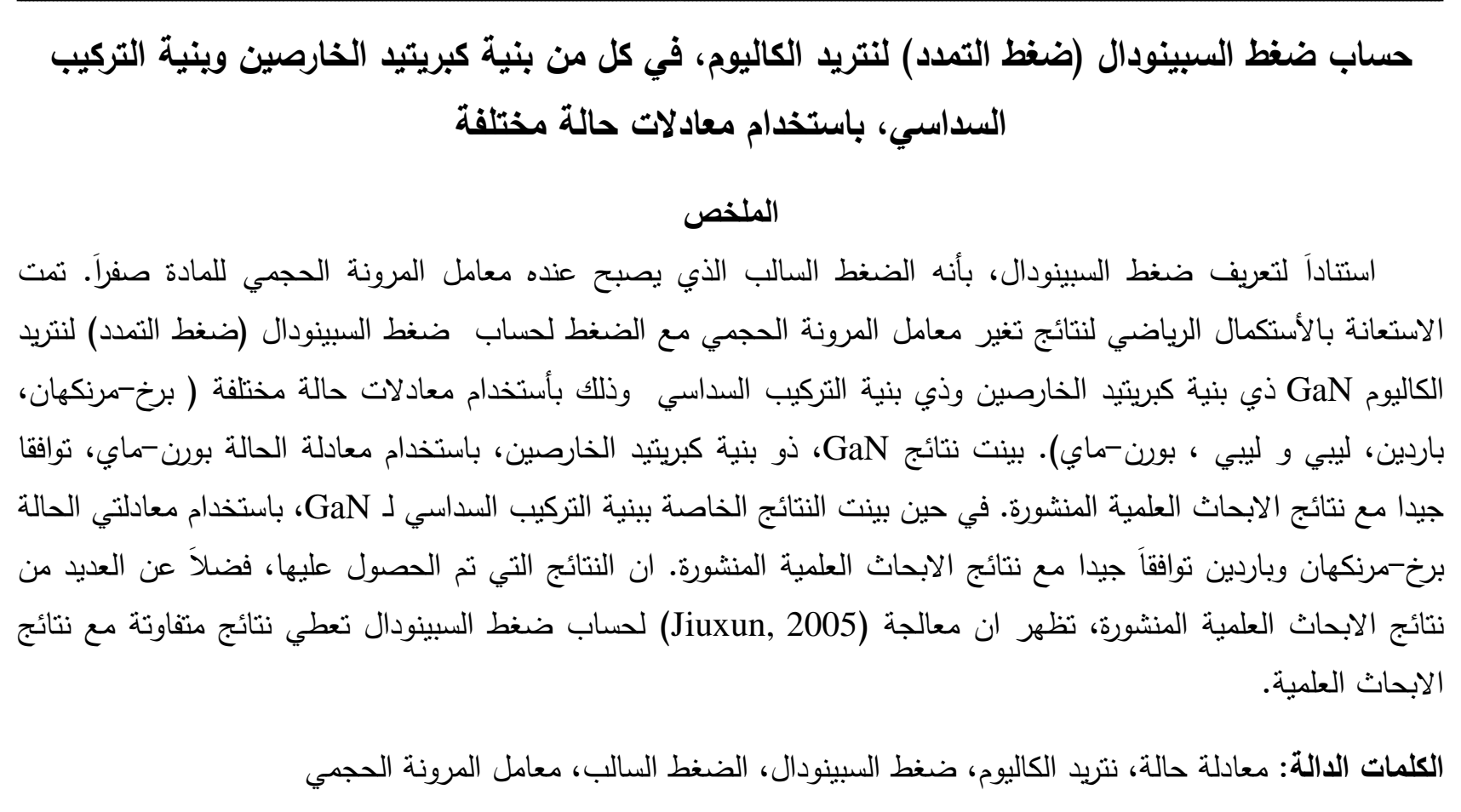

\section{INTRODUCTION}

The calculation of the properties such as enthalpy, entropy, internal energy, compression volume, etc for different substances requires knowledge of the PVT (pressure-volume-temperature) behavior of the substance. This can be given in the form of a table, graphs, or analytically, an analytical expression to express PVT behavior which is called an equation of state. The correct equation of state (EOS) is the gateway to obtaining spinodal and other derived properties, which are 
then used to develop corresponding states correlations. It is sufficient to consider the PVT equation of state (Shamsundar and Lienhard, 1993).

There are at least two conditions which should be satisfied by an equation of state in order to be physically acceptable, these conditions are (Singh and Gajendra, 2011):

(i) The pressure derivative of bulk modulus $(\mathrm{dB} / \mathrm{dP})$ in the limit of extreme compression $\quad(\mathrm{V} \rightarrow 0$ and $\mathrm{P} \rightarrow \infty$ ) must be greater than $5 / 3$.

(ii) Real and reasonable values of properties should be obtained for the entire range of compressions.

In the following we write out the EOS considered in this work, which they have these merits and also satisfy the following spinodal condition (Jiuxun, 2005).

$$
B \propto\left(P-P_{s p}\right)^{\frac{1}{2}} \quad \text { with } \quad B\left(P-P_{s p}\right)=0
$$

where $\mathrm{P}$ : pressure. $\quad \mathrm{P}_{\mathrm{sp}}$ : spinodal pressure. $\mathrm{B}$ : the bulk modulus.

- Birch-Murnaghan EOS (Birch, 1952)

$$
P_{B M}=\frac{3 B_{o}}{2}\left(\eta^{-\frac{7}{3}}-\eta^{-\frac{5}{3}}\right)\left[1+\frac{3}{4}\left(B_{o}^{t}-4\right)\left(\eta^{-\frac{2}{3}}-1\right)\right]
$$

- Bardeen EOS (Bardeen, 1938)

$$
P_{B}=3 B_{o}\left(\eta^{-\frac{5}{3}}-\eta^{-\frac{4}{3}}\right)\left[1+\frac{3}{2}\left(B_{o}^{\prime}-3\right)\left(\eta^{-\frac{1}{3}}-1\right)\right]
$$

- Libby and Libby EOS (Libby and Libby, 1972)

$$
P_{L L}=3 B_{o}\left(\eta^{-\frac{5}{3}}-\eta^{-\frac{4}{3}}\right)
$$

- Born-Mie EOS ( Anderson, 1995)

$$
P_{B-M i}=\frac{3 B_{o}}{3 B_{o}^{t}-8}\left(\eta^{-\left(B_{o}^{t}-\frac{4}{3}\right)}-\eta^{-\frac{4}{3}}\right)
$$

where $\eta=\frac{V_{p}}{V_{o}}, B_{o}$ and $B_{o}^{\prime}$ are the bulk modulus and its first derivative at zero pressure, $\mathrm{V}_{\mathrm{o}}$ is the volume at zero pressure, $\mathrm{V}_{\mathrm{p}}$ is the volume at applied pressure $(\mathrm{P})$.

\section{(1) Bulk modulus under high pressure.}

\section{THEORITICAL DETAILS AND RESULTS}

The bulk modulus (B) of a substance measure the substance's resistance to uniform compression. It is defined as the ratio of the infinitesimal pressure increase to the resulting relative decrease of the volume, formally expressed by the equation:

$$
B=-V \frac{d P}{d V}
$$

From above EOSs, we can express the isothermal bulk modulus $\left(\mathrm{B}_{\mathrm{T}}\right)$ at pressure $(\mathrm{P})$ as follows.

- Birch-Murnaghan EOS

$$
B_{T}(B M)=\frac{3 B_{o}}{2}\left[\frac{7}{3} \eta^{-\frac{7}{3}}-\frac{5}{3} \eta^{-\frac{5}{3}}+\frac{9}{4}\left(B_{o}^{\prime}-4\right) \eta^{-3}-\frac{7}{2}\left(B_{o}^{\prime}-4\right) \eta^{-\frac{7}{3}}+\frac{5}{4}\left(B_{o}^{\prime}-4\right) \eta^{-\frac{7}{3}}\right] \ldots
$$

- $\quad$ Bardeen EOS

$$
B_{T}(B)=3 B_{o}\left[\frac{5}{3} \eta^{-\frac{5}{3}}-\frac{4}{3} \eta^{-\frac{4}{3}}+3\left(B_{o}^{\prime}-3\right) \eta^{-2}-5\left(B_{o}^{\prime}-3\right) \eta^{-\frac{5}{3}}+2\left(B_{o}^{\prime}-3\right) \eta^{-\frac{4}{3}}\right]
$$

- $\quad$ Libby and Libby EOS

$$
B_{T}(L-L)=3 B_{o}\left[\frac{5}{3} \eta^{-\frac{5}{3}}-\frac{4}{3} \eta^{-\frac{4}{3}}\right]
$$

- $\quad$ Born-Mie EOS

$$
B_{T}(B-M i)=\frac{B_{o}}{3 B_{o}^{t}-8}\left[\left(3 B_{o}^{t}-4\right) \eta^{-\left(B_{o}^{t}-\frac{4}{3}\right)}-4 \eta^{-\frac{4}{3}}\right]
$$


Fig. (1) and Fig. (2) show the results of variation of thermal bulk modulus $\left(\mathrm{B}_{\mathrm{T}}\right)$ at different values of applied pressure calculated by using equations $(6,7,8$ and 9) for $\mathrm{GaN}$ in zinc-blende structure and in wurtzite structure respectively. The values of $B_{o}$ and $B_{o}^{\prime}$ which have been used in the calculations are tabulated in Table (1).

Table 1: values of $B_{o}$ and $B_{o}^{\prime}$ for GaN (Christensen and Gorczyca, 1994)

\begin{tabular}{|c|c|c|}
\hline material & $B_{o}$ (Gpa) & $B_{o}^{\prime}$ \\
\hline GaN (zinc-blende structure) & 184 & 4.6 \\
GaN (wurtzite structure) & 200 & 3.8 \\
\hline
\end{tabular}

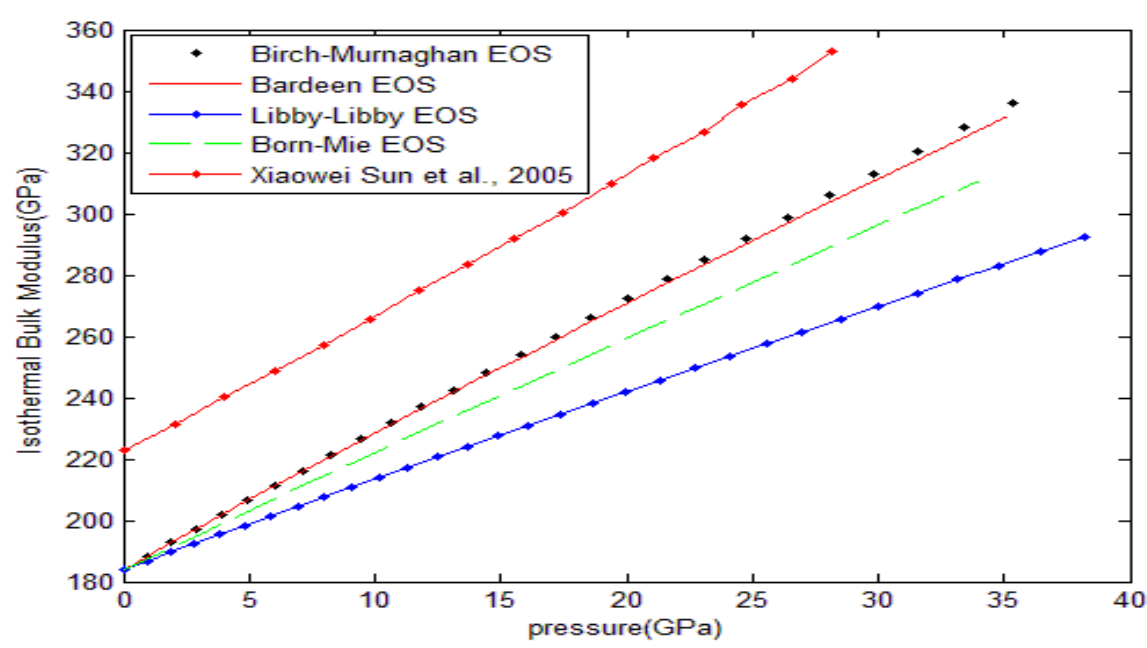

Fig. 1 : Variation if isothermal $\left(B_{T}\right)$ bulk modulus with pressure $(P)$ using different EOS for GaN (zinc-blende structure).

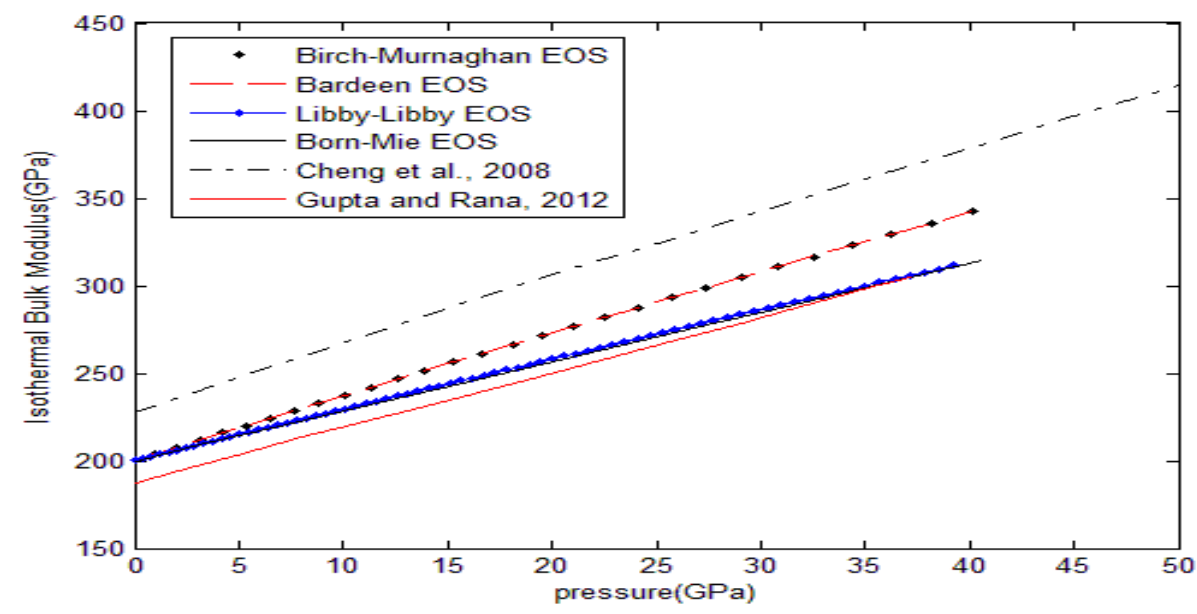

Fig. 2 : Variation of isothermal bulk $\left(B_{T}\right)$ modulus with pressure $(P)$ using different EOS for GaN (wurtzite structure).

(2) The spinodal pressure.

Since the spinodal pressure for material represents the negative pressure at which the bulk modulus of the material goes to zero (Al-sheikh et al., 2013), which whatever its values decreased or in other words its negative values increased no additional expansion occur in material (Stixrude, 2002). By using extrapolating results shown in Fig. (1) and Fig. (2), one can obtain the spinodal 
pressure from the following expression (Al-Sheikh and Mohammed, 2012; Al-Saqa and Al-Sheikh, 2013):

$$
P_{s p}=-B_{o} \frac{d P}{d B}
$$

The values of $\mathrm{P}_{\mathrm{sp}}$ for $\mathrm{GaN}$ (zinc-blende and wurtzite structures) are shown in Fig.(3), Fig. (4) and in Table (II), in comparison with values calculated by equation 11 which was given by (Jiuxun, 2005):

$$
P_{s p}=-\frac{B_{o}}{4 n}, \text { where } n=-\frac{1}{3} B_{o}^{\prime} \quad \therefore P_{s p}=-0.75 \frac{B_{o}}{B_{o}^{\prime}}
$$
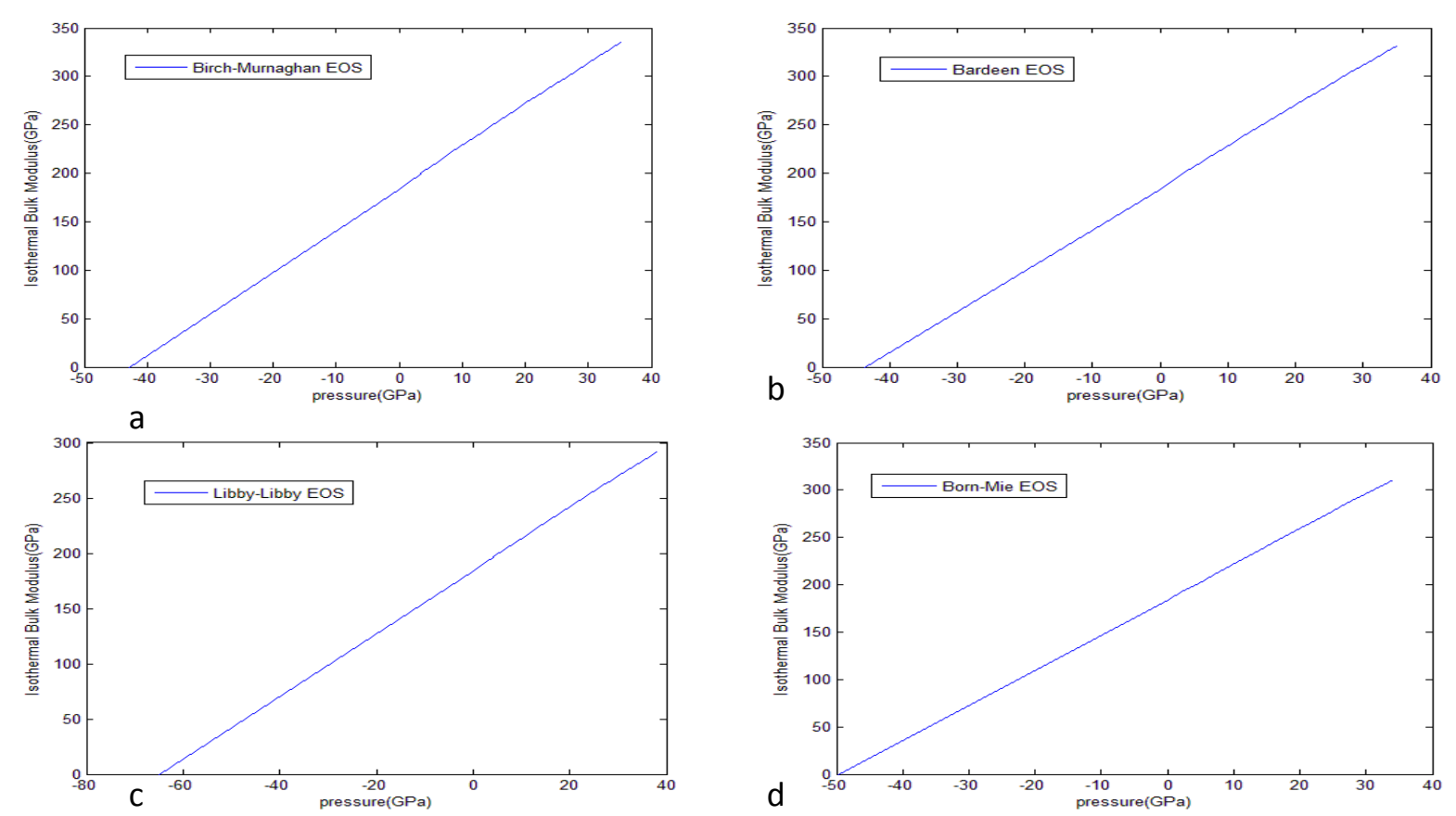

Fig. 3: Spinodal pressure for GaN (zinc-blende structure) by using (a) BirchMurnaghan EOS (b) Bardeen EOS (c) Libby and Libby EOS (d) Born-Mie EOS
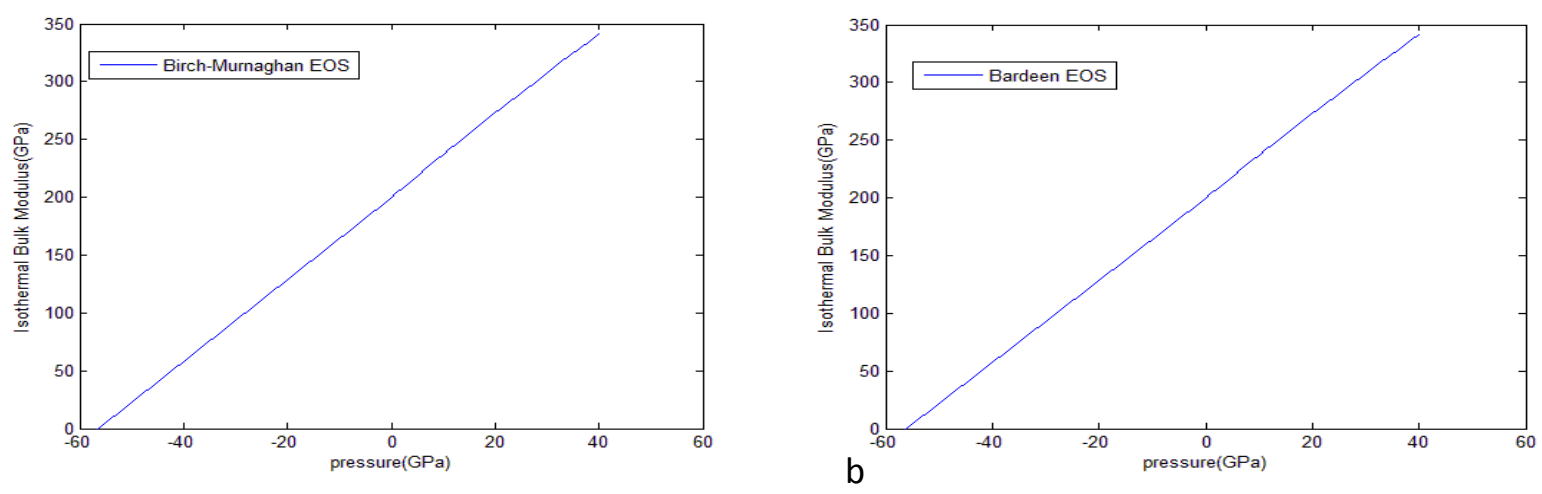

a 

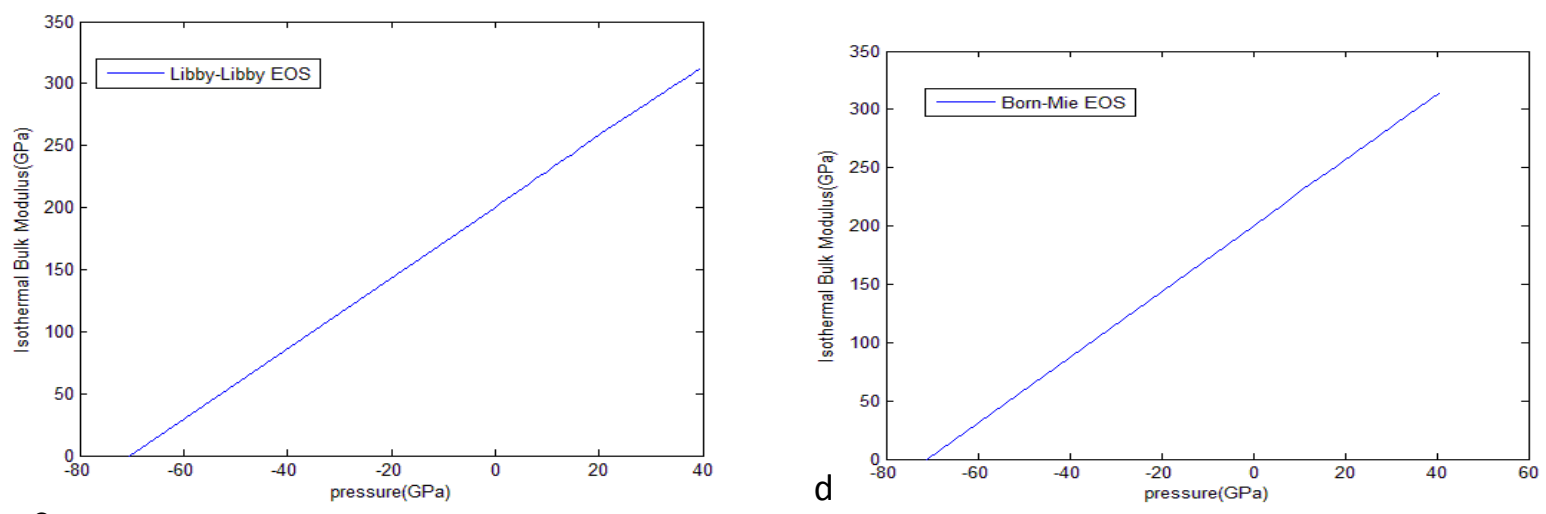

Fig. 4: Spinodal pressure for GaN (wurtzite structure) by using (a) Birch-Murnaghan EOS (b) Bardeen EOS (c) Libby and Libby EOS (d) Born-Mie EOS

Table 2: Spinodal pressure values in comparison with results given in literatures

\begin{tabular}{|c|c|c|c|c|c|c|}
\hline \multirow{2}{*}{ material } & \multicolumn{2}{|c|}{ Present work } & \multirow{2}{*}{$\begin{array}{c}\text { Jiuxun, } \\
2005 \\
\text { Psp(Gpa) } \\
\end{array}$} & \multirow{2}{*}{$\begin{array}{l}\text { Xiaowei } \\
\text { et al., } \\
2005 \\
\text { Psp(Gpa) } \\
\end{array}$} & \multirow{2}{*}{$\begin{array}{r}\text { Gupta and } \\
\text { Rana, 2012 } \\
\text { Psp(Gpa) }\end{array}$} & \multirow{2}{*}{$\begin{array}{c}\text { Cheng } \\
\text { et al., 2008 } \\
\text { Psp(Gpa) } \\
\end{array}$} \\
\hline & EOS & Psp(Gpa) & & & & \\
\hline $\begin{array}{c}\text { Zinc-blende } \\
\text { GaN }\end{array}$ & $\begin{array}{l}\text { Birch-Murnaghan } \\
\text { Bardeen } \\
\text { Libby and Libby } \\
\text { Born-Mie }\end{array}$ & $\begin{array}{r}-42.847 \\
-43.766 \\
-64.943 \\
-49.6975 \\
\end{array}$ & -30 & -48.3564 & & \\
\hline $\begin{array}{c}\text { Wurtzite } \\
\text { GaN }\end{array}$ & $\begin{array}{c}\text { Birch-Murnaghan } \\
\text { Bardeen } \\
\text { Libby and Libby } \\
\text { Born-Mie }\end{array}$ & $\begin{array}{l}-56.4775 \\
-56.36 \\
-70.4523 \\
-71.053\end{array}$ & -39.4737 & & -59.0739 & -60.9647 \\
\hline
\end{tabular}

\section{DISCUSSION}

Experimental results are meager and quite hard to obtain particularly in the neighborhood of spinodal limit, because spinodal is the limit of metastability of substance with respect to phase transition, therefore we used extrapolation of bulk modulus data under high pressure shown in Fig. (1) and Fig. (2), combining these data with equation 5, and (depending on) the definition of spinodal pressure as the negative pressure at which bulk modulus value go to zero. Equation 10 represent a new approach for evaluating spinodal pressure. By using different EOSs which satisfy spinodal condition. The spinodal pressure values for $\mathrm{GaN}$ obtained in present work is close together with results obtained from works of (Cheng et al., 2008), and (Gupta and Rana, 2012), but differ from that obtained by (Jiuxun, 2005), this may be attributed to the fact that our present work used first principle approach in evaluation of $\mathrm{P}_{\mathrm{sp}}$ for material obtained within the metastable state, while (jiuxun, 2005) work didn't consider that (Psp) for the material obtained within the metastable state. Present work show that $\mathrm{P}_{\mathrm{sp}}$ value obtained by Born-Mie EOS is in a good agreement with values given by (Xiaowei et al., 2005) for $\mathrm{GaN}$ in zinc-blende structure. While using, either BirchMuranghan or Bardeen EOS, in the present work, in evaluating $\mathrm{P}_{\mathrm{sp}}$ value for GaN in wurtzite structure, reveal a good agreement with the values given by (Gupta and Rana, 2012) and (Cheng et al., 2008). 


\section{REFERENCES}

Al-Saqa, R.H.; Al-Sheikh, A.M. (2013). Theoretical high pressure study for evaluation of spinodal pressure and phonon frequency spectrum of silver. Raf. J. Sci., 24, 87-95.

Al-Sheikh, A.M.; Mohammed H.B. (2012). Estimation of spinodal pressure by extrapolation of modified general Lennard-Jones equation of state (MGL-J EOS). J. Edu. and Sci.,25, 3, 39-43.

Al-Sheikh, A.M.; Saleh, M.M.; Abdullah, S.J. (2013). Evaluation of spinodal pressure for silicon and germanium using modified Birch-Munaghan and Bardeen equation of state. Raf. J. Sci., 24, 95-99.

Anderson, O.L. (1995). "Equations of State of Solids for Geophysics and Ceramic Science". Oxford University Press, Inc pp. 186- 187.

Bardeen, J.J. (1938). Compressibility's of the alkali metals. Chem. Phys., 6, 372.

Birch, F. (1952). Elasticity and constitution of the earth's interior. Geophys. Res., 57, 227.

Cheng, Y.; Tu, Y.; Zeng, Z.; Gou, Q. (2008). Shell model for elastic and thermodynamic properties of gallium nitride with hexagonal wurtzite structure. Commun. Theor. Phys., 50, 1443-1448.

Christensen, N.E.; Gorczyca, I. (1994). Optical and structural properties of III-V nitrides under pressure. Phys. Rev. B, 50, 4397-4415.

Gupta, D.C.; Rana, P. (2012). Study of semiconducting nanomaterials under pressure. J. Mol. Model, 18, 3341-3350.

Jiuxun, S. (2005). A modified Lennard-Jones-type equation of state for solids strictly satisfying the spinodal condition. J. Phys. Condens. Matter, 17, L103-L111.

Libby, L.M.; Libby, W.F. (1972). One-parameter equation of state for metals and other solids. Proc. Nat. Acad. Sci. USA, 69, 3305-3306.

Shamsundar, N.; Lienhard, J.H. (1993). Equations of state and spinodal lines- a review. Nuclear Engineering and Design, 141, 269-287.

Singh, B.P.; Gajendra, S. (2011). Analysis of compatibility of the generalized Rydberg equation. Indian J. of pure and Appl. Phys., 49, 467-469.

Stixrude, L. (2002). Talc under tension and compression spinodal instability, elasticity, and structure. J. Geophys. Res., 107, 2327.

Xiaowei, S.; Qifeng C.; Yandong C.; Chengwei W. (2005). Structural and thermodynamic properties of GaN at high pressures and high temperatures. Physica B, 368, 243-250. 